\title{
Zwischen Organisation und Verfahren: Rechtskommunikation in der vormodernen Gesellschaft
}

Bevor die Genese des RKG nachgezeichnet wird, geht es darum, ein historisches Verständnis über die gesellschaftlichen Strukturbedingungen der vormodernen Rechtskommunikation zu gewinnen. Leitend ist dabei die Frage, inwiefern sich vor der Gründung des RKG und während seines Bestehens kollektive Erwartungszusammenhänge im Sinne organisatorischer Vorkehrungen und Ausgangslagen beobachten lassen: Inwiefern orientierte sich die Rechtskommunikation an personenübergreifenden Rollen und programmatischen Entscheidungsregeln? Der Fokus auf diese Identifikationspunkte sachlich generalisierter Verhaltenserwartungen ist mit der Prämisse verknüpft, dass Änderungen in der Erwartungsbildung von Werten und Personen hin zu Rollen und Programmen Hinweise auf einen Zuwachs an gesellschaftlicher Komplexität in Richtung Organisationsbildung und funktionaler Differenzierung geben können (siehe Abschn. 3.3.3).

Zentral ist an dieser Stelle, dass das Alte Reich kein Rechtsstaat im ,modernen" Sinne war, sondern ein Personenverband, in dem Herrschaft durch persönliche Kontakte in der Kommunikation unter Anwesenden ausgeübt wurde. Die Könige und Kaiser reisten mit ihren Hof von einer Pfalz zur anderen und kontrollierten so das Reich von wechselnden Orten aus. Je nach Region setzte sich der Hofstaat aus anderen adligen Personen zusammen, die sich dem Reichsoberhaupt während seiner Reisen anschlossen (siehe zum „Reisekönigtum" Aretin 1983). Meine These in diesem Kapitel ist, dass sich demgegenüber bereits vor der Einrichtung des RKG funktional spezifizierte Erwartungszusammenhänge und kollektive Entscheidungskontexte in Richtung Rechtsetzung (vgl. Luhmann 1972a, 152 f., 182 f., 218 ff.), Rechtsprechung (vgl. ebd., 182 f., 173 f.) und Rechtsdurchsetzung (vgl. ebd., 267) ausmachen lassen. Dabei argumentiere ich, dass sich im Übergang vom Spätmittelalter hin zur Frühen Neuzeit

R. Schwarting, Organisationsbildung und gesellschaftliche Differenzierung,

Organisation und Gesellschaft - Forschung,

https://doi.org/10.1007/978-3-658-32872-6_5 
gewisse Änderungen in der Schwerpunktsetzung sachlich-generalisierter Verhaltenserwartungen vollzogen haben, die allerdings innerhalb der stratifizierten Gesellschaft limitiert waren. Der Rückgriff auf die Unterscheidung von Rechtsetzung, Rechtsprechung und Rechtsdurchsetzung als funktionalen Spezifikationen rechtlicher Konfliktkommunikation ist hier ein Behelf, mit dem eingerechnet wird, dass die Ausdifferenzierung von funktionalen Teilsystemen für das Spätmittelalter und die Vormoderne nicht fraglos angenommen werden kann. ${ }^{1}$ Zur empirischen Plausibilisierung dieses Arguments veranschauliche ich zunächst am Beispiel spätmittelalterlicher Stadtgerichte des 14. Jahrhunderts - und damit für die Zeit vor der Gründung des RKG -, dass die gesellschaftlichen Erwartungen an die Rechtsetzung, Rechtsprechung und Rechtsdurchsetzung weitgehend zusammenfielen (Abschn. 5.1).

In einem zweiten Schritt (5.2) verdeutliche ich in dia- und synchroner Perspektive, wie sich die Rechtskommunikation in der Umwelt des RKG teilweise funktional in Richtung Rechtsetzung, Rechtsprechung und Rechtsdurchsetzung spezifizierte. Als kollektive Erwartungs- und Entscheidungszusammenhänge greife ich beispielhaft drei überregionale Einrichtungen auf: Dazu zählen das Reichshofgericht (1235-1451) und das Königliche Kammergericht (1415-1495) (Abschn. 5.2.1) sowie die Hoftage ab dem 12. Jahrhundert bzw. die späteren Reichsversammlungen ab dem 15. Jahrhundert (Abschn. 5.2.2). An diesen Kontexten lässt sich erkennen, wie Entscheidungen über die Rechtsprechung und Rechtsetzung im Reich - zumindest unterstellbar - vollzogen und als solche inszeniert wurden. Als kollektive Erwartungskontexte der Rechtsdurchsetzung können zudem die Policeyordnungen und deren lokale Anwendungen (ab ca. 1450) verstanden werden. Mit dieser allmählichen Differenzierung der Rechtskommunikation und dem damit verbundenen Einsatz von Funktionseliten, deuten sich zwar gewisse Schwerpunktverlagerungen in der sachlichen Generalisierung der Verhaltenserwartungen hin zu Rollen und Programmen an. Als formal organisiert lassen sich die Strukturbedingungen in den ausgewählten Kontexten der Rechtsetzung und Rechtsdurchsetzung jedoch nicht beschreiben. Vor dem Hintergrund der personen- und herkunftsgeprägten Rechtsetzung und Rechtsdurchsetzung erscheint der Blick auf die Strukturbedingungen in der Rechtsprechung vor der

\footnotetext{
${ }^{1}$ Mit der vorgeschlagenen Unterscheidung von Rechtsetzung, Rechtsprechung und Rechtsdurchsetzung ist zugleich die Binnendifferenzierung des politischen Systems angesprochen (siehe Luhmann 2010; 1987b). Im Band „Legitimation durch Verfahren“ (1983) findet sich diese Trias zudem in den Kapitelüberschriften „Politische Wahl und Gesetzgebung“, „Gerichtsverfahren“ und „Entscheidungsprozesse in der Verwaltung“ wieder.
} 
Gründung des RKG (Abschn. 5.3) umso aufschlussreicher für das Verständnis der gerichtlichen Organisationsbildung am Ende des 15. Jahrhunderts (Kap. 6). ${ }^{2}$

Hinsichtlich des Verhältnisses von Theorie und Empirie besteht in den folgenden Abschnitten die Herausforderung, dass die Einsichten aus der historischen Forschung vor der Gründung des RKG in Anbetracht des begrenzten Umfangs dieser Arbeit nur generalisiert und verkürzt dargestellt werden können. Aufgrund des eingeschränkten Analysefokus lässt sich nicht ausreichend beurteilen, ob und inwiefern im Einzelnen oder in anderen Bereichen nicht auch Verhaltenserwartungen auftraten, in denen von gesellschaftlichen Status- und Rangfragen zugunsten formal organisierter Verfahrens- und Mitgliedsrollen abgesehen wurde.

\subsection{Rechtserwartungen im (Spät-)Mittelalter: Zusammenfallen von Rechtsetzung, Rechtsprechung und Rechtsdurchsetzung}

Wie bereits angesprochen, können die gesellschaftlichen Strukturvoraussetzungen von Organisationsbildungen - wie die Ausbildung von Geldwirtschaft, die Gewährleistung von Vertrags-, Eigentums- und Assoziationsfreiheit sowie ein Erziehungssystem, das soziale Aus- und Weiterbildungsrollen ermöglicht (siehe Abschn. 3.3.1) - für die Gesellschaft im Alten Reich nicht ohne Weiteres vorausgesetzt werden. Charakteristisch für das zeitgenössische Rechtserleben waren unterschiedlich institutionalisierte und konkurrierende Rechtsnormen (siehe auch Luhmann 1999b, 308), die in der historischen Forschung insbesondere unter dem Begriff der „Rechtsvielfalt“ (Oestmann 2002, 2011) gefasst werden. Die Rechtskommunikation beruhte dabei in erster Linie auf Tradition, Gewohnheit und faktischem Herkommen und nur zu einem geringen Teil auf positiven, schriftlich gesatzten Normen (vgl. Stollberg-Rilinger 2013a, 13; 2014b, 204; Luhmann 1972a, 130 ff., 183 ff.). Das Reichsrecht enthielt zwar „Freiräume und Schutzzusagen auch zugunsten der mittelbaren Untertanen von Kaiser und Reich“ (Weitzel 1993, 177), jedoch kein übergreifendes Gleichheitsgebot in Form von (reichs-)bürgerlichen Freiheitsrechten bzw. subjektiven Rechten. Beispielsweise

\footnotetext{
${ }^{2}$ In literarischer Form veranschaulicht beispielsweise die Erzählung „Michael Kohlhaas“ von Heinrich von Kleist (2008 [1810]), dass in der Frühen Neuzeit Rechtsetzung und Rechtsprechung keine Rechtsdurchsetzung sicherten. Einsichten aus der belletristischen Literatur und deren Auswertungen können sich für eine historisch-soziologische Organisationsforschung als instruktiv erweisen. Neben den Arbeiten von Kleist lassen sich hier die Werke von Kafka, Balzac, Storm, Fontane usw. nennen.
} 
wurden rechtswidrige Handlungen von adligen Würdenträgern gegenüber rangniederen Personen anders beurteilt als im umgekehrten Fall. Neben der hohen Vielfalt unterschiedlich institutionalisierter Rechtsnormen (insbesondere göttlicher und weltlicher Natur) kann das Fehlen eines zentralisierten und politisch legitimierten Gewaltmonopols als Ausdruck dafür gesehen werden, dass die Bedingungen der Rechtskommunikation nicht mit denen eines modernen, souveränen Wohlfahrtsstaats vergleichbar waren. Mangels geeigneter Exekutionsinstanzen konnten Urteile oft nicht vollstreckt werden. Rechtsprechung, Rechtsetzung und Rechtsdurchsetzung waren weitgehend prekär und kaum auf änderbare Entscheidungsstrukturen zurückführbar (vgl. Weitzel 1993, 157 ff.; Troßbach 2010, 65 ff.; 2009, 85; Luhmann 1970, 321 ff.; 1972a, 328 ff.).

Meine These vor diesem Hintergrund ist, dass Rechtsbehauptungen im Spätmittelalter durch ein Zusammenfallen von Erwartungen der Rechtsetzung, Rechtsprechung und Rechtsdurchsetzung geprägt waren. Rechtliche Strukturbildungen waren dabei nicht als gesonderte Entscheidungsverfahren von einer gesellschaftlichen Umwelt abgetrennt, sondern orientierten sich vielmehr an den in dieser hervorgehobenen Personen und Werten. ${ }^{3}$ Sie spiegelten in diesem Sinne gleichsam die ständisch-hierarchischen Strukturen der ,guten Gesellschaft“ wider (vgl. Luhmann 1987b; Kieserling 2001).

Wie lässt sich dies genauer vorstellen? Zum Verständnis der zentralen Strukturaspekte rechtsförmiger Erwartungszusammenhänge im (Spät-)Mittelalter gilt es zu vergegenwärtigen, dass Rechtsstreitigkeiten weitgehend im Freien auf prinzipiell für alle zugänglichen Plätzen entschieden wurden (vgl. Arlinghaus 2004a/b, 2005, 2006). Was als Recht galt, wurde durch Rituale der Selbstjustiz in der Interaktion unter Anwesenden bestimmt. Insbesondere der Zweikampf diente der Konfliktlösung. Das Duell gab dem archaischen Rechtsverständnis einer maßlosen Vergeltung zwar eine gewisse physische Begrenzung. Zugleich war der Zweikampf Ausdruck eines gottesgerichtlichen Beweismittels, bei dem juristische Sachverhalte auf die einfache Formel von „Mann gegen Mann“ gebracht und nach dem Talionsprinzip „Auge um Auge“ gehandhabt wurden. Die Drastik der Sanktionen einer auf Blutrache und Zweikampf angelegten Rechtskommunikation blockierte mithin die Ausformulierung von standes- und regionsübergreifenden

\footnotetext{
${ }^{3}$ In der Geschichtswissenschaft ist es insbesondere den Arbeiten über die „Herstellung und Darstellung von Entscheidungen“ (Stollberg-Rilinger/Krischer 2010; siehe auch Krischer 2014) zu verdanken, Luhmanns Verfahrenstheorie für vormoderne Fälle fruchtbar gemacht zu machen. Die Beiträge liefern empirische Einsichten, die von der Verfahrens- und Organisationssoziologie zu ihrem Nachteil nicht aufgegriffen wurden (siehe demgegenüber Heintz 2014).
} 
Rechtsvorschriften (vgl. Luhmann 1972a, 145 ff.; siehe auch Hüppe-Dröge 1984, $620 \mathrm{f}$.).

Angesichts der sozialen Kosten, die mit der Praxis der Selbstjustiz verbunden waren, kam zunehmend ein Bedarf an sachlicheren Wegen der Konfliktbearbeitung auf (vgl. Luhmann 1972a, 158 f.). Duellierungen wurden dabei zunächst auf bestimmte Personenkreise beschränkt. Insbesondere dem König war der Zweikampf aufgrund der ihm zugeschriebenen Würde untersagt. Als Reichsoberhaupt war er ,oberster Richter und Hüter von Frieden und Recht“, und von seiner Autorität wurde jedes Urteil abgeleitet. Bereits die ronkalischen Gesetze von 1158 fixierten, dass alle Gerichtsgewalt beim Herrscher liege und dass Richter ihr Amt vom König empfangen mussten. Entsprechend fiel dem Reichsoberhaupt mit seiner Anwesenheit die örtliche Gerichtsbarkeit zu (vgl. Scheurmann 1994, 17 ff.; Diestelkamp 1999, 265, 375; Oestmann 2010, 52 f.). Zur Ausführung der Rechtsprechung standen ihm dabei drei Möglichkeiten offen: Erstens konnte er im Rahmen der königlichen Banngewalt Gebote oder Verbote aussprechen und sich unverbindlich von seinen Urteilern beraten lassen. Zweitens konnte er Reichsstände mit der lokalen Urteilssprechung beauftragen. Drittens hatte er die Option, einen Prozess vor dem Hofgericht entscheiden zu lassen. Ähnlich konnten auch Fürsten Prozesse in ihrem Territorium an sich ziehen und Urteile aufheben (vgl. Diestelkamp 1994a, 111 f.; 1999, 265 f., 325; Schildt 2006, 8).

Ausdrücklich verboten war der Zweikampf nicht nur dem obersten Herrscher, sondern auch nicht waffenfähigen Geistlichen sowie Alten, Frauen, Kranken und Juden. Für diese Personengruppen bestand die Möglichkeit einer Stellvertretung insbesondere durch Freunde und Verwandte, aber auch durch kampferprobte Freie (vgl. Hüppe-Dröge 1984, 633). Neben dem Zweikampf waren nach der karolingischen Zivilgerichtsbarkeit bis ins 13. Jahrhundert ,die Zuschauer unabdingbarer Teil des Gerichthaltens“ (Vismann 2011, 133). „Freie Männer“ bildeten dabei den sogenannten „Umstand“, der ein „Urteil schelten oder mit einem Gegenurteil angreifen konnte" (ebd.). ${ }^{4}$

Eine der zentralen Autoritätslinien in dieser Zeit war die Trennung von weltlichen und geistlichen Gerichten. Die Zuständigkeitsgrenzen zwischen diesen waren dabei weniger vom Rechtsgegenstand abhängig als von der Ausbildung der Richter und der Hoheit des Gerichts (vgl. Oestmann 2012, 6 ff.). Der Einbau

\footnotetext{
${ }^{4}$ Ein ,umstandsloses“ Verfahren (in camera) war dagegen im kanonischen Prozess vorgesehen, auf den bei der Gründung des RKG zurückgegriffen wurde (siehe Abschn. 5.3 und Kap. 6). Wie gezeigt werden wird, wurde dabei in geschlossenen Räumen getagt und über Urteile ohne Umstände schriftlich entschieden. Aus den sogenannten Umständen in Form konkreter Personen wurden vielmehr „sächliche Umstände“ und „bloße Zuschauer“ (vgl. Vismann 2011, 133).
} 
zeitlicher und sozialer Differenzen in der rechtlichen Konfliktbearbeitung lässt sich am Beispiel der spätmittelalterlichen Stadt- und Zunftgerichte beobachten (Arlinghaus 2004a/b, 2005, 2006, 2018). In Köln gab es in fast jedem Kaufhaus ein eigenes Gericht, dem der Bürgermeister vorstand. Aber auch die Angehörigen der Colleges und der späteren Universitäten unterstanden nicht ausschließlich den örtlichen Institutionen, sondern hatten ihre eigene Gerichtsbarkeit. Die Rechtskommunikation und insbesondere die Zuständigkeit war dabei schichtspezifisch differenziert (vgl. Stichweh 1987, 1991; Arlinghaus 2004a, 130 f.; Füssel 2020). ${ }^{5}$

Auch das, was als Recht und Gericht verstanden wurde, war stark durch die jeweiligen gruppen- und standesbezogenen Erwartungen gefärbt. Wie Arlinghaus (2004a, 141 f.) herausgestellt hat, verfügten die Stadtgerichte im Spätmittelalter über keinen eigenen Amtsraum. Wenn eigene Räumlichkeiten genutzt wurden wie insbesondere in Rathäusern -, wurden diese nicht mit dem Gericht assoziiert. Das Mobiliar musste meist eigens aufgestellt werden und die Sitzordnung war nach dem ständischen Rang der Personen gegliedert. In der Gerichtsverhandlung machten v. a. Sprechformeln und das Aufnehmen eines Gerichtsstabes die ritualisierten Eigenheiten der Interaktion sichtbar. Eine Rollentrennung von Richtern und Streitparteien war dabei nicht differenziert. Weder waren die Prozessgegner in eine verfahrenseigene (Zuschauer-)Rolle - selbstbezüglich - ,verstrickt“ (Luhmann 1983, 44; siehe auch Scheffer et al. 2008), noch wurde von den ,Richtern' nach gesatzten Rechtsnormen entschieden, die auf einen Konflikt anzuwenden gewesen wären. Von den Urteilern wurde vielmehr anhand des jeweiligen Streitfalls und durch Fragen an die Gerichtsgemeinde das konkretisiert, was die Rechtsgemeinschaft - zu der auch die Streitparteien gerechnet wurden - zur Beilegung dieses Konflikts für richtig hielt. ${ }^{6}$ Faktisches Handeln wurde dabei dem moralisch-richtigen Handeln gegenübergestellt und ein Konsens über das jeweilige Recht hergestellt.

\footnotetext{
${ }^{5}$ Die deutschen Universitäten des späten Mittelalters besaßen als privilegierte Personenverbände die ,uneingeschränkte Zivilgerichtsbarkeit“ über ihre Angehörigen. Im reichsweiten Vergleich werden mithin erhebliche Unterschiede in der konkreten Ausgestaltung der jeweiligen Gerichtskompetenzen erkennbar, welche insbesondere die Abhängigkeiten von den Gründungsgewalten - Kirche, Landesherren oder Städte - widerspiegeln. Während allerdings zur Gerichtsbarkeit der meisten Hochschulen einzelne Aufsätze vorliegen, halten sich Monografien und vergleichende Studien bislang in einem überschaubarem Rahmen (vgl. Füssel 2020, 265; siehe auch Füssel 2006).

${ }^{6}$ Gerade bei Gerichtsentscheidungen durch Gottesurteile - oder später durch Folter erzwungene Geständnisse - gab es seitens der Gerichtsgemeinde kaum Möglichkeiten der Mitwirkung am Zustandekommen einer Entscheidung (vgl. Diestelkamp 1999, 263).
} 
Im Allgemeinen war das Richteramt ein Laienamt. Die Urteiler bzw. Beisitzer garantierten lediglich den korrekten Ablauf der Urteilsfindung und deren Durchsetzung: „Ob eine Person nun an der Ratssitzung teilnahm oder als Richter eine Verhandlung leitete, $[\ldots]$ beide Aufgaben übte er [...] als Angehöriger des Patriziats aus; beide Rollen wurden in das Elite-Sein integriert" (Arlinghaus 2004a, 137). Dabei zeigten bestimmte Rituale an, dass die Führungspersonen in den örtlichen Gremien bei der Übernahme der Richtertätigkeit ihre gesellschaftliche Rolle nicht verließen. Die Übergänge zwischen politischen und administrativen Aufgaben waren dabei fließend. Auch die gewohnte Tracht - das maßgebliche Insigne für die Standeszugehörigkeit - wurde beibehalten. Die Ratsrichter einer Stadt waren zugleich auch die Ratsherren. Gelehrte Juristen befanden sich zwar bereits im 13. Jahrhundert unter den Stadtschreibern und Syndici. Zu städtischen Richtern wurden die ausgebildeten Juristen jedoch nicht ernannt. Vielmehr gingen sie in den Dienst der Kirche oder der Landesherren (vgl. Scheurmann 1994, 119; Diestelkamp 1999, 263 f.; Jahns 2011, 70 f., 184; Arlinghaus 2004a, 136 f., 2018, 38 ff.).

Sichtbar wurde das Fehlen verfahrenseigener Rollen insbesondere dann, wenn sich durch ein Urteil nicht nur eine Person, sondern ganze Zünfte oder Gilden kollektiv in ihrer Ehre verletzt sahen und dies öffentlich artikulierten (vgl. Arlinghaus 2004b, 138 ff.). Dabei zeigte sich, dass die Durchsetzung der Urteile tatsächlich „,in der Hand der Partei, die obsiegte“ (Diestelkamp 1999, 264), lag. Auch der Gebrauch von Schrift blieb performativ und beschränkte sich weitgehend auf das Verlesen von Texten. Selbst Amtsträger hatten nur einen restriktiven Zugang zu schriftlichen Texten (vgl. Arlinghaus 2004a, 136 f.; Schlögl 2014a, 10 ff.; 2014b, 193). Angesichts des „Zwangs zur Serialität“ bei Face-to-Face Interaktionen (Luhmann 1972b, 55; 1975a, 11; Kieserling 1999, 40) war die Rechtskommunikation insgesamt zeitintensiv. Formen der Abwicklung von konfligierenden Erwartungen, die eine strukturell höhere Abstraktion und Detailliertheit realisierten, differenzierten sich dagegen erst mit der Einrichtung von (organisierten) Verfahren als entscheidungsförmigen Interaktionssystemen (vgl. Luhmann 1972a, 158 f.; 2014, 24 f.; Schwarting 2017a/b).

\subsection{Funktionale Spezifizierung von Rechtserwartungen}

Im Vergleich zur spätmittelalterlichen Konfliktregelung unter Anwesenden lässt sich in der Frühen Neuzeit ab dem 15. Jahrhundert eine gewisse funktionale Spezifizierung der Rechtskommunikation beobachten. Strukturverschiebungen in der rechtlichen Konfliktbearbeitung der vormodernen Gesellschaft zeigen sich 
darin, dass kollektive Erwartungszusammenhänge unabhängiger von der Interaktion unter Anwesenden wurden und situationsweise ein regelorientiertes Verhalten möglich war. Dabei ist anzunehmen, dass im Einzelnen auch Entscheidungen zustande kamen, die von der ständischen Rangordnung abstrahierten. Jenseits religiöser Gleichheitsansprüche waren Beurteilung, Kritik und Kontrolle des Verhaltens insgesamt jedoch auf den Status einer Person bezogen.

Vor diesem Hintergrund zeige ich erstens hinsichtlich der sachlichen Verhaltensgeneralisierung in der Rechtsetzung auf, dass sich am Reichstag trotz sogenannter Umfragen und der Entsendung von Vertretern noch keine rechtsförmigen, autonomen Verfahren ausdifferenzierten (Abschn. 5.2.1). Die Rechtsdurchsetzung war zweitens auf schichtspezifische Strukturvorgaben beschränkt sowie durch starke Überlappungen in den Zuständigkeiten geprägt (Abschn. 5.2.2). Im Anschluss an die Einsichten in die frühneuzeitliche Rechtsetzung und Rechtsdurchsetzung veranschauliche ich drittens am Beispiel des Reichshofgerichts (1235-1451) und späteren Königlichen Kammergerichts (1415-1495), dass sich die Rechtsprechung vor der Gründung des RKG ebenfalls weitgehend an der Anwesenheit der Person des Königs orientierte (Abschn. 5.2.3). Im Vergleich zur punktuellen Rechtsprechung im Rahmen des Reisekönigtums verdeutlichen insbesondere die Einrichtung eines Hofrichteramtes zur Vertretung des Königs sowie die Einführung von Kanzleiregeln zur Dokumentation von Urteilsentscheidungen einen Zuwachs an struktureller Komplexität. Insgesamt vollzogen sich auf der Ebene der Rechtsetzung, Rechtsprechung und Rechtsdurchsetzung zwar gewisse Schwerpunktverlagerungen in der Erwartungsbildung. Dennoch waren Rechtsbehauptungen, so lassen sich die historischen Forschungen in diesen Bereichen knapp zusammenfassen, durch die Unbedingtheit ständisch-hierarchischer Strukturvorgaben eingehegt.

\subsubsection{Rechtsetzung auf den Reichstagen: Regieren ohne Opposition}

Auf der Ebene der Rechtsetzung können die Ständeversammlungen als zentrale rechtliche Erwartungskontexte genannt werden, die sich im Spätmittelalter in vielen europäischen Ländern in ähnlicher Weise herausbildeten. Empirisch bedeutsam sind die Reichstage deshalb, weil sie einen „Bedarf für Entscheidungen über kollektive Aktion“ (Luhmann 1978a, 44; siehe Abschn. 3.3.2) - insbesondere über Entscheidungen zur Gestaltung der Reichsverfassung - zum Ausdruck bringen. Dabei ist zu erinnern, dass in der stratifizierten Gesellschaft 
des Alten Reichs die Normbestände weitgehend unveränderlich waren. Bemerkenswert ist allerdings, dass sich trotz oder gerade wegen des Rechtspluralismus mit den sogenannten Reichsabschieden auf den Reichsversammlungen Anteile einer varianten Rechtsstruktur, und damit eines kontingenten gesatzten Rechts beobachten lassen. Vor dem Hintergrund dieser Teilpositivierung besaß das Alte Reich keine einheitliche Verfassung, sondern vielmehr eine Reihe von „Grundgesetzen“ (Stollberg-Rilinger 2006, 41), wie etwa den Ewigen Landfrieden von 1495, den Augsburger Religionsfrieden von 1555 oder den Westfälischen Frieden von 1648. Letzterer band den Kaiser in übergreifenden Angelegenheiten ausdrücklich an die Übereinkunft aller Reichsstände auf der Reichsversammlung (vgl. Stollberg-Rilinger 2013b, 101; 2006, 42 ff.).

Insbesondere für die Formulierung ordnungspolitischer Rahmenvorgaben spielte der Reichstag eine zentrale Rolle. So wurden beispielsweise Verbote für Gotteslästerung, Kleiderordnungen oder Bestimmungen zum Zins und zur Warenqualität beschlossen (vgl. Sikora 2004, 176; Härter 2010, 30 ff.). Eine „rechtsvereinheitlichende Integration“ wird in der Frühe-Neuzeit-Forschung insbesondere „wirtschaftlichen Projekten“ wie der „Reichsmünzordnung“ zugesprochen (vgl. Sikora 2004, 176). Zugleich enthielten einige Reichstagbeschlüsse wie die nachstehend behandelte Reichspoliceyordnung sogenannte salvatorische Klauseln. Diese ließen eine Anpassung der reichsrechtlichen Normen an das lokale Recht oder ergänzende partikulare Institutionalisierungen $\mathrm{zu}$, die jedoch das Reichsrecht nicht verletzen durften (vgl. Härter 2006, 92).

Dem Übergang der Reichsversammlung von unregelmäßigen und vom Kaiser einberufenen Treffen zum permanent tagenden Immerwährenden Reichstag ging eine Vielzahl monarchischer Hoftage und Kurfürstenversammlungen voraus. ${ }^{7}$ Der Reichstag unterschied sich von einem mittelalterlichen Hoftag vor allem durch einen „rechtlich verfestigten Teilnehmerkreis“ (Stollberg-Rilinger 2016, 142). Da der Kaiser zunehmend auf finanzielle und militärische Ressourcen seitens der Stände angewiesen war, konnte er immer weniger ad hoc über die Zusammensetzung des Reichstags befinden. Die Stände nutzen diesen Umstand, um sich bestimmte Privilegien und Freiheiten kollektiv verbriefen zu lassen. Dazu gehörte auch das Partizipationsrecht auf der Ständeversammlung. Vor allem die Inhaber

\footnotetext{
${ }^{7}$ Die spätmittelalterlichen Hoftage waren in unregelmäßigen Abständen und an wechselnden Orten vom Reichsoberhaupt quasi privat einberufene Versammlungen. Zunächst nahmen ausgewählte Fürsten - später auch Vertreter der Reichsstädte - an den Hoftagen teil. Die Einberufung von Reichsständen zu den Hoftagen wurde lehnsrechtlich mit dem Treueverhältnis begründet, das den Vasallen als Lehnsherrn gegenüber dem Reichsoberhaupt zu „Rat und Tat" (,consilium et auxilium“) verpflichtete (vgl. Stollberg-Rilinger 2016, 139 ff.; Isenmann 1989; Moraw 1980; 1989).
} 
großer Reichslehen (Kurfürsten, Fürsten, Herzöge, Grafen sowie die Reichsprälaten) erhielten mit der Einrichtung des Reichstages im Rahmen der Reichsreform von 1495 ein persönliches Partizipationsrecht und erbliche Sitze. Ab dem Jahr 1654 setzte der Erwerb der Reichsstandschaft auch den Besitz eines reichsunmittelbaren Territoriums voraus, das als Lehen direkt vom Kaiser übertragen wurde. Das Reich lässt sich vor diesem Hintergrund als ein Interessenverband von Landeigentümern verstehen. Von dem zeitgenössischen Rechtsgelehrten Justus Möser wurde der Reichstag denn auch als eine „Aktienkompanie“ bezeichnet, in der nur mitstimmen dürfe, wer Anteil am „Kapital“ des Reichs besaß (vgl. Schröder 1995; siehe auch Walther 2014).

Die Reichsversammlung umfasste in der Regel mehr als 300 Teilnehmer, deren Stimmen ständisch abgestuft auf drei sogenannte Kurien (Kurfürstenrat, Fürstenrat und Städterat) verteilt waren. Im Unterschied zur Kuriatstimme (Stimme, die von einer Kurie bzw. ,je Bank' gemeinsam abgegeben wurde) war eine Virilstimme (Einzelstimme eines Stimmberechtigen) nur den Kurfürsten und Reichsfürsten vorbehalten (vgl. Kotulla 2008, 20; Stollberg-Rilinger 2006, 42 ff.). Zum Ende des Alten Reichs im Jahr 1792 zählten beispielsweise die kurfürstlichen Stimmen acht, die fürstlichen kamen auf 100 und die reichsstädtische Kurie umfasste 51 Stimmen. Zu den Kurfürsten gehörten neben den geistlichen Bischöfen von Mainz, Köln und Trier auch die weltlichen Herrscher von Böhmen, Sachsen, Brandenburg und der Pfalz. Als fürstliche Vertreter nahmen adlige Standespersonen - meist Vasallen der Krone oder Würdenträger der Kirche - teil. Die ritterlichen Inhaber der kleinen Reichslehen bildeten ab Mitte des 16. Jahrhunderts sogenannte Ritterorte, die zu drei Ritterkreisen zusammengefasst wurden. Weder die Untertanen noch die (nicht am Reichstag teilnehmenden) Angehörigen des niederen Adels konnten für sich ein Mitspracherecht reklamieren. Einzelne Herrschaftsträger schlossen sich, um ihre Interessen zumindest kollektiv zu vertreten, zu Korporationen zusammen. Darunter waren vor allem Städte, aber auch Grafschaften, ländliche Amtsbezirke und geistliche Stifte, die auf dem Reichstag „hinter einer (buchstäblichen Standes-)Schranke stehen bleiben“ mussten (vgl. Stollberg-Rilinger 2016, 141 f.; Sikora 2004, 164; Härter 1997, 191 ff.).

Für das Verständnis über die Rechtsetzung unter den Bedingungen von Anwesenheit ist relevant, dass sich nach der Einrichtung einer ständigen Reichsversammlung die Reichsstände zunehmend durch beauftragte Personen vertreten ließen. Wohlgemerkt handelte es sich dabei nicht um freie Mandate. Die Gesandten waren vielmehr an die Weisungen des jeweiligen Herrschaftsträgers gebunden. Insbesondere die kleineren Reichsstände - wie Grafen, Prälaten, Fürsten und Reichsstädte - schickten seltener eigene Gesandte zum Reichstag und übertrugen ihre Rechte vielmehr an Regensburger Bürger. Die Stellvertretung durch 
bevollmächtigte Gesandte sollte allerdings die Ausnahme sein und die persönliche Präsenz die Regel, durch die die Repräsentation und Bindungskraft von Beschlüssen für das ganze Reich (einschließlich der Abwesenden, Dissentierenden und Untertanen) hergestellt und zugleich symbolisch durch gewohnheitliche Herrschaftsrituale dargestellt wurde. Der Reichstag wurde dabei in seiner hierarchisch gegliederten Form erst in Realpräsenz sichtbar - und dies trotz der Tatsache, dass auf dem Immerwährenden Reichstag ab der zweiten Hälfte des 17. Jahrhunderts auch Kaiser und Fürsten nicht mehr in Person aufeinandertrafen, sondern ihre Gesandten (vgl. Stollberg-Rilinger 2013b, 104 f.; 2014b, 204 f.; 2016, 138 f.; Krischer 2009, 193).

Dass die Gesandten weitgehend unter sich waren, ist erklärungsbedürftig. Für viele Reichsstände hatten die lokalen Verpflichtungen Priorität vor den oft langwierigen und ergebnislosen Versammlungen. Gerade den Fürsten bot der eigene Hof effektvollere Bühnen zur Selbstdarstellung und Machtdemonstration. Viele Herrscher schickten deshalb Personen an den Reichstag, die sie geringschätzten und entsprechend unterbezahlten, denn die Kosten der Stellvertretung überstiegen aus ihrer Sicht oft den Nutzen der Partizipationsrechte. Zwar beauftragte Bremen beispielsweise bis zum Ende des Reichs durchgehend Regensburger Ratsherren mit der Vertretung seiner Stimme. Für deren Tätigkeit aber hat es sich offenbar kaum interessiert: Die Reichstagspost wurde häufig noch ungeöffnet ins Archiv weitergeleitet (vgl. Krischer 2012, 10). Dies war nicht zuletzt auch darin begründet, dass die Stimme der Reichsstädte von den Kurfürsten und Fürsten weitgehend ignoriert wurde, ,indem sie die Städte erst und nur dann um ihr Votum fragten, wenn sie sich unter einander geeinigt hatten" (Stollberg-Rilinger 2013b, 106).

Mit der standesungleichen Repräsentation waren vor allem im zeremoniellen Umgang vielfältige Rangkonflikte und Statusfragen verknüpft. Diese äußerten sich darin, dass sich die Gesandten untereinander nicht mit den Würden des jeweiligen bevollmächtigenden Herrschers bzw. Reichsstandes begegneten, weil diese in ihrem Rang nicht ebenbürtig waren. Während sich beispielsweise die adligen Vasallen nicht ausweisen mussten, hatten Deputierte der Städte ihre Berechtigung zuerst durch Beglaubigungsschreiben zu belegen. In der fehlenden Akzeptanz der Selbstdarstellung durch Dritte wird gesellschaftstheoretisch deutlich, dass Vertretungsrollen auf der Reichsversammlung nicht standesübergreifend übernommen werden konnten und personengebunden waren. Die mangelnde Anerkennung rangniederer Gesandter hatte zudem einen negativen Selbstverstärkungseffekt. Denn auch die kleineren Fürsten interessierten sich im Verlauf des 17. und 18. Jahrhunderts immer weniger für die kollektive Repräsentation des Reichsverbandes. Dem „Gesandtenkongress“ (Stollberg-Rilinger 2013b, 107) wird denn auch 
neben seiner ,politischen“ Funktion eine latente Bedeutung als „Nachrichtenumschlagplatz“ und „Knotenpunkt informeller Netzwerke“ zugeschrieben (ebd., 109.; siehe auch 2014b, 211 f.; 2016, 142 ff.; Krischer 2009, 2012).

Die Dominanz einer personenbezogenen Ranghierarchie bei der Rechtsetzung zeigt sich zudem im Eid - dem sogenannten Antrittskompliment -, mit dem die Gesandten einen ,heiligen Bund“ zwischen Reichsoberhaupt und Reichsgliedern eingingen. Wie Stollberg-Rilinger (2013b, 110) herausstellt, waren die im Antrittskompliment formulierten Pflichten kaum in einer Rolle einheitlich darstellbar: Die Gesandten verpflichteten sich nicht nur zur Ehrerbietung gegenüber dem Reichsoberhaupt. Von Eigennutz und Willkür befreit, hatten sie darüber hinaus sowohl der Hoheit und Wohlfahrt des gesamten Reichs zu dienen als auch zugleich die Rechte des jeweiligen Fürstenstandes und seiner Religion zu vertreten. Die damit verbundenen Ansprüche gegenüber dem obersten Herrscher, dem Reich als Ganzem und dem Reichsstand waren jedoch nicht identisch. Sie standen vielmehr in einem Werte- und Interessenkonflikt (vgl. Stollberg-Rilinger 2001, 15; 2013b, 110).

Der Abstimmungsprozess am Reichstag war gewohnheitsrechtlich abgeleitet: Die feierliche Eröffnungssitzung wurde zunächst als Bitte des Herrschers ,um Rat und Hilfe der Stände zum Wohle des ganzen Gemeinwesens“ (Stollberg-Rilinger 2016, 146) inszeniert. Der Monarch gab dabei die Agenda der jeweiligen Reichsversammlung in Form einer feierlichen Proposition und Thronrede vor. Dem sogenannten Direktor des Reichstages kam die Aufgabe zu, das vom Kaiser vorgestellte Beratungsthema in eine Frageform zu überführen, zu der die Stimmvoten nach ständischer Rangfolge abzugeben waren. Das jeweilige Thema wurde dann von den drei Kurien separat durch innerkuriale Beratungen und konsensuale Beschlussfassung in sogenannten Umfragen behandelt. $\mathrm{Zu}$ jedem Beratungspunkt gaben die Teilnehmer der Reihe nach ihr Votum ab. Es wurde so lange reihum gefragt, bis sich eine konsensfähige Meinung herauskristallisiert hatte. Anschließend wurde die Stellungnahme dann interkurial zwischen Kurfürsten und Reichsständen verglichen, bis zwischen diesen ein Konsens formulierbar war. Da eine Minderheitenposition tendenziell als unwahr, unrecht und unmoralisch galt, hielten sich viele Gesandte zu Beginn mit ihren Stimmen zunächst zurück, um abzuwarten, wie andere votierten. Das Ergebnis wurde am Ende den Vertretern der Städte mündlich verkündet, ohne dass deren Einwände zwingend Berücksichtigung fanden. Der Kaiser wiederum konnte abweichende Positionen zur Geltung bringen, die in erneuten Abstimmungsvorgängen mit den ständischen Vorstellungen in Einklang zu bringen waren. Weder eine einzelne Kurie noch der Kaiser konnten überstimmt werden. Wesentlich war dabei, dass Beratung und Abstimmung nicht getrennt waren, sondern fließend ineinander übergingen. Was 
als Ergebnis zu verstehen war, beruhte nicht auf einer Gleichheit der Stimmen, sondern richtete sich nach den sozialen Statusverhältnissen der Beteiligten (vgl. Sikora 2004, 168 ff.; Stollberg-Rilinger 2016, 139 ff.; Krischer 2009, 185 ff.).

Wie André Krischer (vgl. 2009, 191) festhält, konnte dies beispielsweise zur Folge haben, dass der Reichstagsdirektor in seinem Bericht ein Votum erklärte, das nur von einem oder wenigen, aber ständisch gewichtigen Beteiligten vertreten wurde. Wie auch immer das Ergebnis der Umfrage faktisch erzielt worden war - ob durch offene Gespräche oder geheime Absprachen -, es wurde in den Protokollen und gegenüber den anderen Reichstagsgesandten stets so dargestellt, als ob es das Resultat einer gemeinsamen (göttlichen) Wahrheitssuche im Rahmen der offiziellen und den Konsens symbolisierenden Umfragen gewesen wäre. Weniger der offene Ausgang einer Entscheidung als vielmehr die Institutionalisierung dieser Einigkeits- bzw. Einheitsfiktion galt damit in der Tradition des europäischen Ständewesens als Ausweis der gegenseitigen Verbindlichkeit. Das Umfrageprinzip unterband vor diesem Hintergrund eine sichtbare „Herrschaft des Verfahrens“ (Krischer 2009, 191).

Eine Trennung von Diskurs und Quantifizierung der Stimmen und eine - nicht zuletzt durch politische Wahl - änderbare Differenz von Regierung und Opposition wie in modernen Parlamenten gab es damit auf dem Reichstag nicht. ${ }^{8}$ Offene Konflikte wurden vielmehr außerhalb der Sitzungen - oft an einem Tisch in den sogenannten Nebenzimmern unter ,Suspendierung ihrer Rangposition und Privilegien“ (Krischer 2009, 204) - verhandelt. Die Beschlussfassung wurde schließlich schriftlich als Reichsabschied (und mit der Einsetzung des Immerwährenden Reichstags ab 1663 als Reichsgutachten) dem Kaiser zur Ratifizierung vorgelegt. Erst mit seiner Zustimmung konnte ein Beschluss verabschiedet werden (siehe auch Sikora 2004, $167 \mathrm{f}$.).

Zum Verständnis der Strukturbedingungen, unter denen die Rechtsetzung auf den Reichstagen standfand, ist nicht zuletzt relevant, dass den Reichsständen ein breites Repertoire an Möglichkeiten zur Einflussnahme zur Verfügung stand (z. B. Anfragen, Änderungen der Proposition, Mahnungen, Verzögerungen, Nebenverhandlungen, Audienzen oder Vorträge kaiserlicher Kommissare). Faktisch wurden am Ende jedoch meist die Erwartungen des Monarchen (im Hinblick auf Geldzahlungen) erfüllt. Die Stände konnten zwar Bitten vortragen, aber das Ansinnen des Herrschers nicht einfach ablehnen, ohne dass damit die gesellschaftlichen Normen der Anerkennung einer ständischen Hierarchie gebrochen worden wären.

\footnotetext{
${ }^{8}$ Entsprechend überrascht es, wenn in den (rechts-)historischen Forschungen von „Regierung“ oder „Gesetzgeber“" gesprochen wird. Das Fehlen einer theoretischen Rückversicherung der verwendeten Begrifflichkeiten setzt die Hürden bei ihrer soziologischen Übersetzung damit relativ hoch (siehe Abschn. 4.1).
} 
Umgekehrt legten die Stände Wert darauf, dass sie ihre Voten frei getroffen hätten und dass diese Entscheidungen als solche verstanden wurden. Denn an der unterstellten freiwilligen Beteiligung hing reichsrechtlich ihr Status als Partizipationsberechtigte des Reichstags und von diesem wiederum waren ihre Rechte und Privilegien im Reich abhängig (vgl. Krischer 2009, 184 ff.; Stollberg-Rilinger 2016, 148 f.; Härter 2006, 82 ff.).

In der Zusammenschau der Rechtsetzungsstrukturen fällt es angesichts des Primats von gesellschaftsübergreifenden Konsensvorstellungen sowie des Fehlens einer personenunabhängigen Rollendifferenzierung und damit verbundener Selbstbindungseffekte schwer, von einer „Legitimation durch Verfahren“ (Luhmann 1983) auszugehen. Es gab keine Themenautonomie, keine formale Geschäftsordnung und auch der Teilnehmerkreis wurde persönlich vom Reichsoberhaupt bestimmt. Aus den historischen Forschungen lässt sich vielmehr ein Verständnis von Rechtsetzung in Form von Aushandlungen ableiten, bei dem die Kondensierung einer Entscheidung weitgehend nach versäulten Konfliktlinien verlief. Von Versäulung spricht Luhmann in Anlehnung an Lipset (1962, 1967a/b; siehe auch Kruijt/Goddijn 1965; Vanderstraeten 1999), wenn Interessenkonflikte gesellschaftsweite Erwartungsdifferenzen abbilden (vgl. 1972a, 248; 2002, 133). Symptomatisch für die fehlende Isolierung der Entscheidungsfindung von gesellschaftlichen - insbesondere ständischen und konfessionellen - Spannungen und Problemlagen war nicht zuletzt, dass die Verhandlungen auf den Reichsversammlungen oftmals scheiterten.

\subsubsection{Rechtsdurchsetzung in Form „guter Policey“: Rechtsunsicherheit und Kompetenzüberlagerung}

Neben der Rechtsetzung auf den Reichstagen lassen sich mit den Reichspoliceyordnungen kollektive Erwartungskontexte für eine Rechtsdurchsetzung festmachen. Auch hier ist zu betonen, dass sich die Strukturbedingungen der Durchsetzung von Rechtsnormen im Alten Reich mit dem Modell eines modernen, durch Polizeiorgane exekutierten Verwaltungsverständnisses nicht vergleichen lassen. Wie oben angesprochen, war die Rechtsdurchsetzung insgesamt durch Rechtspluralismus und Rechtsunsicherheit gekennzeichnet. Zur Durchsetzung seines Jurisdiktionsanspruchs setzte der Herrscher neben dem Hofgericht (siehe Abschn. 5.2.3) im 14. und verstärkt im 15. Jahrhundert sogenannte königliche Kommissare ein oder delegierte Aufgaben an persönliche Vertreter (vgl. Mitsch 2003). Zugleich wurden zahlreiche Verwaltungsaufgaben im Spätmittelalter von kommunalen und genossenschaftlichen Einrichtungen ausgeübt. Demgegenüber 
lassen sich seit dem 16. Jahrhundert unter dem Begriff „gute Policey“ anwachsende Ordnungsansprüche in der ständischen Herrschaftsverwaltung beobachten, die einen Prozess der Säkularisierung über obrigkeitliche Normsetzung markieren (vgl. Blickle/Schüpbach 2003; Härter 2010, 2013). Manifest wurden die reichsständischen Bestrebungen nach Autonomie insbesondere in der Verabschiedung des Ewigen Landfriedens auf dem Reichstag von 1495.

Zur Durchsetzung des Reformwerks, auf dem auch die Gründung des RKG beschlossen wurde (siehe Abschn. 5.3), wurde die Wahrung von „Ordnung und Policey“ in den Zuständigkeitsbereich der lokalen Herrschaftsträger verlagert. Gegenstand der Reichspoliceyordnung waren insbesondere krisenhafte überregionale Problemlagen (z. B. Bevölkerungszunahme, Teuerung, Hunger, Seuchen, Fehde und Gewalttaten), die von den Obrigkeiten als Missstände und Unordnung angesehen wurden. Entsprechend dominierten Regelungen zum Kredit- und Münzwesen, zu Handel und Handwerk sowie zur öffentlichen Sicherheit im Allgemeinen - nicht zuletzt zur Wahrung des Landfriedens beim Umgang mit Aufständen und Unruhen (vgl. Härter 2019, 348 f.).

Die Reichspoliceyordnungen ermächtigten die Reichsstände auch zum Erlass eigener partikularer Gesetze. Hinsichtlich der Policeyordnung der Reichsstadt Frankfurt am Main verzeichnen Karl Härter und Michael Stolleis (2004) in ihrem Repertorienband insgesamt 5.057 Beschlüsse für die Zeit von 1329 bis 1806. Die Verteilung der einzelnen Normen im Zeitverlauf verdeutlicht dabei die steigende Bedeutung der öffentlichen Sicherheit, wobei vor allem das 18. Jahrhundert den höchsten Anstieg verzeichnete. Inhaltlich manifestierte sich diese Entwicklung insbesondere im Hinblick auf Eigentumsdelikte durch Raub- und Diebesbanden sowie auf politisch motivierte Verbrechen (vgl. 2010, 246 f., 255 f.).

Die plurale und fragmentierte Normsetzung des Alten Reichs wies dabei im Strafrecht die größten Mängel und Unsicherheiten auf (vgl. Westphal/Härter 2013, 665): Weder waren Verbrechen eindeutig fixiert noch die dafür jeweils angedrohten Strafen. Auch die Rechtsdurchsetzung war prekär, bedingt nicht zuletzt durch fehlende Rechtsmittel. Die Aufgabe der Urteilsvollstreckung war seit 1512 den Reichskreisen übertragen worden, zu denen im 16. Jahrhundert die verschiedenen Reichsstände einer Region unter der Leitung eines oder mehrerer Fürsten zusammengelegt worden waren. Aufgrund dieser dezentralen Verlagerung etablierten sich keine eigenen Policeyorgane zur reichsweiten Durchsetzung der Policeyordnungen. Die Reichsinstitutionen wandten die Reichspoliceyordnungen ,nur gelegentlich an“ (Härter 2019, 349). Insbesondere bei Prozessen gegen Reichsstände war die Vollstreckung eine Machtfrage. Auf territorialer Ebene unterstanden die Reichsstände zwar einer gewissen Kontrolle, denn Verstöße gegen das Reichsrecht konnten sowohl durch andere Reichsstände als auch durch Untertanen vor die Reichsgerichte gebracht werden (vgl. Härter 2006, 92 f.). Die 
Landesherren konnten sich der Umsetzung der Reichsnormen jedoch leicht entziehen und schufen bei Konflikten nicht selten selbst Fakten, um zu ihrem Recht zu gelangen - sei es beispielsweise über die Vernichtung von Beweismitteln oder das Foltern von Aufständischen (vgl. Sabean 1982; Bähr 2011, 2012a/b).

Umgekehrt versuchten auch Kirche, Zünfte, Gemeinden sowie zu Diensten verpflichtete Bürger, zusätzliche policeyliche Aufgaben zu umgehen. Sie hatten dabei einen vergleichsweise hohen Spielraum, denn die lokalen Einrichtungen waren allgemein überlastet und konnten nicht zuletzt aufgrund der geringen finanziellen Zuwendungen seitens der Territorialherren nicht ausgebaut werden. Die Ordnungsarbeiten lokaler Herrschaftsträger und Einrichtungen wurden dadurch weiter eingeschränkt. Auch handelte es sich bei den Ausführenden der Verwaltungsarbeit um Laien, die keine professionelle Ausbildung an einer Universität absolviert, sondern durch ihre Zugehörigkeit zum lokalen Patriziat und Bürgertum ein Erfahrungswissen gewonnen hatten. Der Einfluss der Reichspoliceyordnungen blieb dadurch auf die lokale Verwaltungstätigkeit begrenzt. Die entsprechenden herrschaftlichen Einrichtungen übten bei geringer Arbeitsteilung und funktionaler Spezialisierung praktisch Justiz- und Verwaltungsfunktionen zugleich aus - mit der Folge, dass zahlreiche Kompetenzkonflikte entstanden und die Zuständigkeiten häufig wechselten. Die lokalen Herrschaftsträger mussten z. B. neben der erstinstanzlichen Zivilgerichtsbarkeit auch in inquisitorischen Strafverfahren sowie in der Frevel- und Rügegerichtsbarkeit Aufgaben übernehmen (vgl. Stolleis 2000, 739 ff.; Härter 2010, 251 ff., 263 f.; Raphael 2000, 26 ff.).

Nach 1648 konnten sich die Reichsstände immer seltener auf gemeinsame Policeynormen einigen. Der Immerwährende Reichstag verabschiedete zwar zwischen 1666 und 1676 zu einzelnen Bereichen noch Entwürfe und Reichsgutachten. Diese führten jedoch zu keiner neuen Reichspoliceyordnung. Im 18. Jahrhundert ging daher die Anzahl der Policeybeschlüsse des Reiches zurück. Die Reichsmitglieder erließen vielmehr eine wachsende Zahl partikularer Ordnungen, die spezifisch für das jeweilige Herrschaftsgebiet einzelne Bereiche des Policeywesens reglementierten (vgl. Diestelkamp 1999, 210 f., 260 ff.; Jahns 1990, 59; 2011, 83 f.; Sikora 2004, 167; Stollberg-Rilinger 2013b, 109 f.).

Insgesamt blieben die Erwartungs- und Entscheidungszusammenhänge der Rechtsdurchsetzung in ihren Bindungseffekten und ihrer funktionalen Spezifizierung diffus, unsicher und fragmentiert. Auch wenn der Normenpluralismus im Alten Reich kaum Rechtssicherheit zuließ, sondern materielle Unvollständigkeit und Unüberschaubarkeit des Rechts prägend waren, wird den Policeyordnungen eine zentrale Funktion bezüglich der Normierung und Professionalisierung der Verwaltungstätigkeiten zugeschrieben. Die historische Forschung betont in dieser Hinsicht, dass sich gerade für die Endphase des Alten Reiches Strukturelemente 
in Richtung eines modernen Rechts ausbildeten. Verwiesen wird dabei auf den aufklärerischen Rechtsdiskurs und die damit verbundenen Bestrebungen zur Vereinheitlichung der Rechtsaufzeichnungen. Diese konzentrierten sich vor allem auf eine strikte Rechtsbindung der Richter, die Verdrängung von Laien, die Beseitigung konkurrierender Gerichtsbarkeiten, die Eliminierung der landesherrlichen Gnadengewalt sowie die Bekanntmachung und Vereinfachung der Ordnungstexte (vgl. Härter 2013, 668 ff.).

\subsubsection{Rechtsprechung am Reichshofgericht und Königlichen Kammergericht: Reichsoberhaupt als oberster Herrscher und Rechtsherr}

Um ein Verständnis über die rechtsförmigen Erwartungs- und Entscheidungskontexte in der vormodernen Gesellschaft zu gewinnen, gilt es neben den Strukturbedingungen der Rechtsetzung und Rechtsdurchsetzung während des Bestehens des RKG auch die der Rechtsprechung vor seiner Gründung zu konturieren. Dabei behandele ich beispielhaft das spätmittelalterliche Reichshofgericht (1235-1451), das im 15. Jahrhundert durch das Königliche Kammergericht (1415-1495) ersetzt wurde.

Wie oben erläutert, war der König nach mittelalterlicher Anschauung „oberster Richter und Hüter von Frieden und Recht“ (Diestelkamp 1999, 265). Alle anderen Richter mussten ihr Amt vom Reichsoberhaupt empfangen. Aus diesem Grundsatz folgte, dass der König jeweils dort, wo er sich aufhielt, Prozese an sich ziehen konnte. Gleiches galt für die territorialen Herrscher, die Streitfälle an ihren Hof verlegen konnten. Im Mittelalter und in der Frühen Neuzeit existierten dazu verschiedene Hofgerichte. Das oberste Gericht war das Reichshofgericht, das mit dem Mainzer Landfrieden von 1235 eingerichtet wurde und an den Aufenthaltsort des Reichsoberhauptes gebunden war. Das Reichshofgericht ist dabei nicht als höchste Instanz im modernen Sinne zu verstehen, denn es gab nicht die Möglichkeit, gegen ein untergerichtliches Urteil Rechtsmittel einzulegen (vgl. Oestmann 2005a/b, 2010).

Als strukturelle Neuerungen des Reichshofgerichts werden in der rechtshistorischen Forschung die Einrichtung eines Hofrichters und einer Hofgerichtskanzlei behandelt. Der Einsatz eines ständigen Hofrichters ging auf die Einschränkungen des Fehderechts und den dadurch gestiegenen Rechtsprechungsbedarf zurück. Vor Beginn einer Fehde musste nunmehr ein Gericht befragt werden, und erst wenn dieses kein Urteil fällte, konnte eine Fehde ausgetragen werden. Der Mainzer Landfrieden hob das Fehderecht damit zwar nicht vollständig auf - dazu kam es 
erst 1495 mit dem Ewigen Landfrieden und der Gründung des RKG -, aber er grenzte es zeitlich, räumlich und sozial ein. Dazu wurden beispielsweise nicht waffenfähige Personen (z. B. Frauen, Bauern, Juden, Kaufleute etc. sowie Kirchen) ausgeschlossen (vgl. Diestelkamp 1994a, 110 ff.). Im Allgemeinen wurden am Reichshofgericht Streitfälle der sogenannten Reichsunmittelbaren entschieden, d. h. von Personen, die direkt dem Kaiser untergeben waren. Dazu zählten Konflikte zwischen Fürsten, Streitigkeiten über Reichsgüter und Reichsrechte oder Fälle territorialgerichtlicher Rechtsverweigerung. Das Reichshofgericht wurde dabei vor allem vom niederen Adel sowie den Städten und ihren Bürgern in Anspruch genommen. Zwischen 1276 und 1451 werden dem Reichshofgericht etwa 18.000 Gerichtsbriefe zugerechnet, von denen 2.000 erhalten sind (vgl. Oestmann 2005b, 162 ff.; 2010, 52).

Am Reichshofgericht übte der genannte Hofrichter den Vorsitz aus. Er war allerdings nicht an der Urteilsfindung im engeren Sinne beteiligt. Der Hofrichter brauchte keine akademische Ausbildung vorzuweisen, sondern qualifizierte sich durch seine Zugehörigkeit zum Reichsadel. Die Urteile wurden von den Beisitzern bzw. Urteilern gefällt. Der König behielt sich die Verhandlung der Klagen von Fürsten wie auch Achturteile vor. Zugleich konnte er weiterhin territoriale Urteilsentscheidungen an sich ziehen. Wie der König war auch der Hofrichter nur dort rechtsprechend tätig, wo er persönlich anwesend war. Obwohl er sein Amt nicht als Lehen verliehen bekam, sondern mit einer Amtsbesoldung übertragen erhielt, endete die Beauftragung des Hofrichters mit dem Tod des Königs (vgl. Diestelkamp 1994a, 111).

Wie der Hofrichter gehörten auch die beratenden Beisitzer des Reichshofgerichts dem Adel an und stammten aus Personenverbänden der ,königsnahen Landschaften“ (Diestelkamp 1999, 266). Die Urteilsfindung unter den Beisitzern erfolgte einvernehmlich im Konsens und auf der Grundlage der bestehenden Rechtsgewohnheiten in mündlicher Form. Der Hofrichter sollte zudem von einer Kanzlei unter Leitung eines Gerichtsschreibers unterstützt werden. Der Hofgerichtsschreiber war ein juristisch geschulter Laie, aber nicht rechtsgelehrt, sondern vielmehr für die Führung der Register und Urteilsbücher zuständig. Im Gegensatz zum Hofgericht wurden für die Hofgerichtskanzlei Angehörige des Niederadels und des Bürgertums als Notare zugelassen, die ebenfalls Laien waren. Sie behielten ihre Ämter auf Lebenszeit (vgl. Diestelkamp 1999, 193 f., 276; Seyboth 1994, 68 ff.). Erwähnenswert ist in diesem Zusammenhang auch, dass in der Hofgerichtskanzlei erste Regeln zur Führung eines schriftlichen Registerwesens entwickelt wurden, mit dem der Beginn des modernen Aktenwesens verbunden wird (siehe dazu Vismann 2010, 164 ff.; Diestelkamp 1995a, 29). 
Angesichts eines anhaltend hohen Bedarfs an Konfliktentscheidungen führte der Monarch neben dem Reichshofgericht ein sogenanntes Königliches Kammergericht ein, das 1451 das Reichshofgericht schließlich ganz ersetzte. Bezeichnet wurden mit dem Königlichen Kammergericht zunächst die immer regelmäßiger gewordenen Beratungen des Königs ,in seiner Kammer durch adlige und gelehrte Räte“ (Diestelkamp 1999, 267), die ab 1415 urkundlich dokumentiert sind. Das Königliche Kammergericht wird in der historischen Forschung noch stärker als das Reichshofgericht als ein Instrument der persönlichen Rechtsprechungsinteressen des Königs und späteren Kaisers Friedrich III. (1415-1493) beschrieben. Die Herrschaftszeit Friedrichs war die längste und reichsfernste der römischdeutschen Oberhaupte: Angesichts langjähriger dynastischer Konflikte verlagerte sich unter ihm der Herrschaftsmittelpunkt von Böhmen in die österreichischen Erblande und damit an die südöstlichen Randgebiete des Reichs. Während seiner über 53-jährigen Herrschaft war er von 1444 bis 1471 nicht im Binnenreich präsent. Um seinen Herrschaftsanspruch aus der Ferne aufrecht zu erhalten, setzte er für unterschiedliche Funktionen Kommissare als Stellvertreter und Mandate ein (vgl. Diestelkamp 1999, 195, 276 f., 393 ff.; Mitsch 2003, 7 ff.; Männl 2010, $331 \mathrm{ff} ., 346 \mathrm{ff}$.).

Durch die langjährige Abwesenheit kam es am Königlichen Kammergericht zum Verfahrensstillstand. Angesichts der unentschiedenen Konflikte im Reich verstärkten sich die Reformbemühungen der Reichsstände. Im Jahre 1461 reagierte der Kaiser im Austausch finanzieller Militärhilfen auf den Einfluss der Reichsstände und der Erzbischöfe. In Form einer Ämterverpachtung erhielt das Gericht in der Person des Kammerrichters erstmals einen ständigen Leiter - zunächst Bischof Ulrich von Passau (1461-1470) sowie anschließend den Mainzer Erzbischof Adolf II. von Nassau (1470-1475). Letzterer führte im Rahmen der Pacht nicht nur das Königliche Kammergericht, sondern auch die Kanzlei. Dabei zeigten sich ,,verstärkte Bemühungen um Schriftlichkeit“" (Magin 2004, 5). Bemerkenswert ist, dass am Königlichen Kammergericht bereits durch den Bischof Ulrich von Passau 1465 ein Sitzungsprotokollbuch eingeführt und 1467 ein erstes Urteilsbuch angelegt wurde. Unter Erzbischof Adolf II. wurden die Urteilsbücher fortgeführt und um ein erstes Aktenarchiv sowie ein Taxregister ergänzt. Auch sorgte dieser dafür, dass das Gericht 1471 mit der "reformacie sins kammerihts“ (Diestelkamp 1999, 197) eine erste schriftlich fixierte und einsehbare Ordnung erhielt. Das Interesse an der „Verstetigung der Arbeit und Verbesserung der Judikatur" (Diestelkamp 1995a, 12) beruhte insbesondere darauf, die Einkünfte aus der Gerichtstätigkeit zu steigern, um nicht zuletzt die hohe Pachtsumme zu refinanzieren. Die Rechte aus der Verpachtung konnten jedoch weiterhin nur dann ausgeübt werden, wenn sich der Kaiser am Hofe aufhielt. Für Zeiten, in denen 
der Kanzler und Kammerrichter nicht am kaiserlichen (wandernden) Hof anwesend war, behielt Friedrich III. das Richteramt. Mit der Verpachtung konnte somit Recht stellvertretend nur in ,naher Abwesenheit' gesprochen werden (vgl. Diestelkamp 1994a, 110 ff.; Smend 1911, 311 ff.; Magin 2004, 5 f.).

Die Aufgaben der Kanzlei gingen über die Schreibarbeit hinaus und umfassten auch politische Beratertätigkeiten (vgl. Vismann 2000, 163 f.). Die Verteilung der Aufgaben verlief dabei „nach einem familialen Vorbild“ (ebd.). Die Kanzlei war „derart mit der Person des Herrschers verwoben“, dass sie „ohne Delegationsprinzip“ (ebd., 166) auskommen musste. Vielmehr behielt sich der Herrscher vor, ,alles domini regis propia letztinstanzlich selbst zu entscheiden“ (ebd., Herv. i. O.). Eine kontinuitätssichernde, verstetigende Funktion hatten die Registerarbeiten damit nicht. Die Reichweite der Verregelung war auf die eigene Amtszeit begrenzt. Auch die wechselnden Residenzen machten es letztlich unmöglich, „Ordnung zu halten“ (ebd., 163). Wenngleich sich Aufgaben- und Regeln verdichteten, bildete das Königliche Kammergericht keine eigenständige Institution. Gegen eine Trennung von Reichshofgericht(skanzlei) und Kammergericht(skanzlei) während der Pachtzeiten spricht insbesondere die „Identität von Kanzler und Kammerrichter" in der Person des Erzbischofs und geistlichen Kürfürsten sowie der Taxregister, der nicht zwischen beiden Gerichten unterschied (vgl. Magin 2004, 6, Fn. 30; siehe auch Diestelkamp 2014; Luger 2015).

Zugleich gelang es im 15. Jahrhundert nicht, das Raubrittertum und das Fehdewesen einzudämmen. Gerade das Fehdewesen legitimierte Kleinkriege zwischen Territorialfürsten, Ritterschaft und Untertanen. Zu einer Verschärfung der Konflikte trug bei, dass während der langjährigen Abwesenheit des Reichsoberhaupts die Urteilstätigkeiten und Ausgaben für den Unterhalt des Kammergerichts ins Stocken gerieten (vgl. Scheurmann 1994, 51 f.; Seyboth 1994, 69). Die Schwächung des Königlichen Kammergerichts setzte sich auch unter dem König und späteren Kaiser Maximilian I. (1459-1519) fort. Dieser galt als „ebenso paranoid wie despotisch“ (Vismann 2010, 168) und duldete „keine Eigenmächtigkeit“ (ebd.). Dies sollte sich mit der Reichsreform 1495 ändern (siehe Abschn. 5.3).

Aufbauend auf den knappen Beschreibungen lässt sich die Einschätzung ableiten, dass die Besetzung von Richter- und Kanzleirollen im 15. Jahrhundert auf traditionellen Herrschaftserwartungen basierte und damit nicht eindeutig zwischen „Gerichtsherrn und Rechtskennern“ (Luhmann 1972a, 219) getrennt wurde. Hinsichtlich der obersten Gerichtstätigkeit muss darüber hinaus die genannte gerichtliche Rechtsvielfalt im Alten Reich berücksichtigt werden. Neben evangelischen Konsistorien und katholischen Offizialatsgerichten gab es eine Vielzahl weltlicher Hofgerichte, Zunftgerichte, Gemeindegerichte und Stadtgerichte. Die 
Zuständigkeitsbereiche der jeweiligen Gerichte waren durch Partikularrecht festgelegt und schwankten demnach von Territorium zu Territorium. Streitfälle über Braugerechtigkeiten und Schuldforderungen konnten beispielsweise auch vor geistliche Gerichte gelangen. Ebenso konnten weltliche Gerichte Konflikte über Pfründe und Kirchenbaulasten behandeln (vgl. Oestmann 2012, 6 f.). Peter Oestmann weist darauf hin, dass sogar normative Quellen bekannt sind, nach denen die geistlichen Gerichte selbst entscheiden konnten, ob eine Sache geistlich oder weltlich war (vgl. 2012, 12). Der Bedarf an ausgebildeten Juristen im Spätmittelalter ging dabei hauptsächlich von geistlichen Gerichten und der Kirche aus. Im ausgehenden 13. Jahrhundert waren weltliche Dienstherren für Juristen dagegen selten, während zu Beginn des 15. Jahrhunderts auch Landesherren und Städte Juristen einsetzten. Ausschlaggebend für die juristische Ausbildung und Anstellung - sei es in geistlichen oder weltlichen Diensten - war fast ausschließlich die Funktion und Beziehung zum jeweiligen Dienstherrn. In der Regel handelte es sich um dieselben Personenkreise, weil der lokale Adel personell mit den umliegenden Dom- und Kollegiatstiften verflochten war (vgl. Scheurmann 1994, 119 f.; Schmutz 2000, 75 ff.).

Auch auf der Seite der Streitparteien standen in der Rechtsprechung weniger sachliche als soziale Gesichtspunkte im Vordergrund. Rechtsfragen im Allgemeinen und gerichtliche Zuständigkeitsentscheidungen im Besonderen waren in der stratifizierten Gesellschaft mit Fragen der Zugehörigkeit verknüpft. Zu welchem gesellschaftlichen Stand und Verband jemand gehörte, war beim Zugang zum Gericht oft selbst Gegenstand der Gerichtstätigkeit (vgl. Arlinghaus 2018, 29 ff.). Aus der historischen Forschung sind zwar verschiedene Fälle überliefert, in denen die Angeklagten unterschiedliche Statuszuordnungen für sich reklamierten, durch die sie sich Vorteile für den Prozessverlauf erhofften. Weil Zunftmitglieder und Gemeindegenossen zugleich auch Bürger einer Stadt waren, konnten beispielsweise mehrere Klagen in der gleichen Sache bei verschiedenen Gerichten eingereicht werden. Dort aber, wo es aufgrund von Mehrfachzugehörigkeiten zu Konflikten kam, wurden diese in Fragen der Hierarchie überführt. Überschneidungen und Unvereinbarkeiten versuchte man damit über gesellschaftliche Rangabstufungen zu lösen. Auf diese Weise konnte daran festgehalten werden, dass einer Person ihrem Rang gemäß ein Platz zukam (vgl. Arlinghaus 2018, 40 f.; Stollberg-Rilinger 2001, 2014b).

Auch wenn nicht jeder Verstoß gegen ein Zunftstatut mit Exklusion - sei es durch Stadtverweis oder gar Todesstrafe - geahndet wurde, und es vielfältige alternative Konfliktregelungen gab (siehe auch Cordes 2015), lässt sich für die Gesellschaft zwischen 1250 und 1500 konstatieren, dass die strukturellen 
Veränderungen im Kern innerhalb der gleichen stratifizierten Differenzierungsform stattfanden: Das Verhältnis von Person, Rechtsbehauptung und Gesellschaft ließ sich damit nicht voneinander trennen. Die Einrichtungen der Rechtsetzung, Rechtsprechung und Rechtsdurchsetzung waren im Allgemeinen nicht an verfahrenseigenen Rollen und organisatorischen Mitgliedschaftsregeln orientiert, sondern an Konsensprinzipien und am Status bzw. der Herkunft einer Person. Der dominierende Fluchtpunkt gesellschaftlicher Zugehörigkeitsfragen schloss nicht aus, dass es bei der Rechtsetzung, -sprechung und -durchsetzung im Einzelnen auch um Fragen des Rechts und der Gerechtigkeit im engeren Sinne ging. Die Erwartungs- und Entscheidungszusammenhänge im Recht selbst, wie Luhmann es abstrakt formuliert, gerieten jedoch noch nicht bereichsübergreifend unter Veränderungsdruck (vgl. 1972a, 90 ff.).

\subsection{Originäre Organisationsbildung: Vom Hofgericht zum Gerichtshof}

Bevor die Verhaltenserwartungen bei der Rechtsprechung am RKG, die für die Organisationsförmigkeit des Gerichts sprechen, im Einzelnen aufgezeigt werden (siehe Kap. 6), sollen die gesellschaftlichen Strukturvoraussetzungen der Organisationsbildung selbst genauer erschlossen werden. Die Ausgangsannahme dieser Arbeit ist, dass mit der Gründung des RKG eine Systemumstellung in der Rechtsprechung von den Bedingungen der Interaktion hin zu einer formalen Organisation stattfand. Aus der Perspektive einer funktionalen Analyse (siehe Abschn. 4.2) ist dabei zu fragen, welcher Problemdruck zum Ende des 15. Jahrhunderts dazu führte, dass Konflikte am Königlichen Kammergericht nicht mehr ausreichend bearbeitet werden konnten. Aus der historischen Literatur lässt sich die Vermutung ableiten, dass angesichts der strukturellen Begrenzungen, die sich aus der Verknüpfung von Rechtsfragen mit gesellschaftlichem Status und physischer Anwesenheit ergaben, der Bedarf an einer gewaltfreien, ständigen und höchstrichterlichen Rechtsprechung immer virulenter wurde.

Mit Blick auf die Unterscheidung von abgeleiteter und originärer Organisationsbildung frage ich, wie sich der skizzierte Problemdruck soziologisch fassen lässt, auf den mit der Gründung einer ,immerwährenden' obersten Rechtsprechung in Form des RKG reagiert wurde. Die Frage, welches gesellschaftliche Problem mit der Gründung einer Organisation gelöst wird, ist wie erwähnt kaum systematisch untersucht worden. Was bedeutet es aber, wenn ein Bedarf für die Lösung eines gesellschaftlichen Problems nicht mehr durch die Kommunikation unter Anwesenden gedeckt wird? 
Mein Eindruck ist, dass mit der genannten Unterscheidung das Verhältnis von organisatorischer und gesellschaftlicher Differenzierung weiter aufgeschlüsselt werden kann als mit den herkömmlichen Mitteln der Organisationsforschung (siehe zu einem kategorialen Strukturbegriff von Organisation Abschn. 2.1.1). Zum Verständnis der Vermutung einer originären Organisationsbildung des RKG lohnt es sich, die Reichsreformdebatte im 15. Jahrhundert noch genauer zu umreißen. Instruktiv sind dafür die Notizen des Bischofs und späteren Kardinals Nikolaus von Kues, der auf dem Basler Konzil 1433/34 zur Lage im Reich notierte: „Es gibt wegen der Sorglosigkeit der Kaiser, die glaubten, sie könnten nur mit Freundlichkeit die Verirrungen heilen und reformieren, keinerlei Sorge um den Nächsten und die Zukunft. Jegliche Ahndung ist verschwunden. Aufrührer werden nicht bestraft. Mit Hilfe ihrer tyrannischen Herrschaft sind viele zu mächtigen Fürsten geworden, während es mit dem Reich bergab ging" (Concordantia Catholica, Buch III, Kapitel 29, Nr. 496, aus dem Lateinischen übersetzt von und zit. nach Weinrich 2001, 177).

Ausgehend vom Basler Konzil spitzten sich die Bestrebungen um eine Reichsreform im Verlauf des 15. Jahrhunderts mehrfach zu. $\mathrm{Zu}$ einem gesteigerten Problemdruck trug nicht nur die Reichsferne Friedrichs III. bei, sondern auch, dass die Pächter des Königlichen Kammergerichtes und Kanzleidirektoren ,starke reformerische Impulse entwickelten“ (Press 1987, 8 ff.), um die Gerichtstätigkeit weiter zu verstetigen. Die aktenmäßige Dokumentation der Rechtsprechung - wie die Einführung von Protokoll- und Urteilsbüchern sowie Registern - lag dabei vor allem im finanziellen Interesse der Pächter bzw. Kanzleileiter. Die Kritik an einer präsenzorientierten Rechtsprechung wurde zunehmend lauter als nach dem Tod des erwähnten Mainzer Erzbischof Adolf II. von Nassau (1474) eine Neuverpachtung zunächst ausblieb. Vor dem Hintergrund des verbreiteten Fehdewesens hatten auch die Reichsstände immer wieder versucht, ihre eigenen Gerichtsbarkeiten zu stärken - nicht zuletzt durch einzelne Landfriedensvereinbarungen (vgl. Diestelkamp 1994a, 110 ff.; Smend 1911, 311 ff.).

Interessant ist an den Vorschlägen für eine Reichsreform und die Verstetigung der Gerichts- und Kanzleitätigkeiten, wie sich aus der römisch-rechtlichen Figur rechtswirksamer Vertretung ein allgemeiner Begriff der repraesentatio identitatis (im Unterschied zu repraesentatio potestatis) entwickelte: „Kein Teil kann das Ganze im Ganzen sein; aber es gibt Teile, die zur Repräsentation des Ganzen im Ganzen befugt und befähigt sind" (Luhmann 1997, 920). Dieser Begründungszusammenhang lässt sich auch für die Errichtung des RKG in Anschlag bringen, die sich auf den Reichstagen im Jahre 1486 konkretisierte. Mit dem Verbot eigenmächtiger Rechtsdurchsetzungen in Form der Fehde avancierte die Idee 
zur Einrichtung eines ständigen Gerichts, das für das Reichsoberhaupt stellvertretend Rechtsprechungsrechte übertragen bekommen sollte. Die Finanzierung und Besetzung des Gerichts sollten geteilt durch Reichsoberhaupt und Reichsstände geleistet werden (vgl. Hausmann 1989, 9; Seyboth 1994, 71 ff.; Diestelkamp 1999, 393 ff.; Jahns 2011, 76 ff.). ${ }^{9}$

Die Vorstellungen einer reformierten Gerichtsbarkeit widersprachen den Erwartungen von König Maximilian I.. Aus den historischen Forschungen lässt sich entnehmen, dass der Habsburger König die Reichsstände vor allem als Mittel ansah, um eigene Herrschaftsinteressen im europäischen Machtgefüge durchzusetzen. Dazu habe er insbesondere die eigene Selbstdarstellung als ,letzter Ritter und ,Vater der Landsknechte" gepflegt. Für ihn sollte das RKG lediglich eine Wiederaufrichtung des alten Königlichen Kammergerichts sein. Seine Zustimmung zu einer Reichsreform wird in der RKG-Forschung denn auch auf das Kalkül zurückgeführt, im Gegenzug militärische und finanzielle Unterstützung zur Erweiterung der Reichsgrenzen erhalten zu können. Entsprechend habe er mit einer Reichsreform die Stärkung der Königlichen Zentralgewalt verbunden und nicht deren Delegation an verschiedene Reichsinstitutionen unter Beteiligung der Reichsstände (vgl. Scheurmann 1994, 53; Seyboth 1994, 73 f.; Moraw 1989, 3-47; Stollberg-Rilinger 2006, 42 ff.).

Vor dem Hintergrund der dualistischen Reichsverfassung mit den beiden Polen Reichsoberhaupt und Reichsglieder wurde die Gründung und Unterhaltung des RKG zum „Maßstab für die Bereitschaft des Kaisers, sich mit dem Reich und seinen Ständen auseinanderzusetzen“ (Baumann 2019a, 333). ${ }^{10} \mathrm{Zu}$ den vielfältigen Einflussnahmen, mit denen auch die nachfolgenden Herrscher versuchten,

\footnotetext{
${ }^{9}$ Zur Erinnerung: Auch wenn man, wie es in dieser Arbeit der Fall ist, annimmt, dass es sich bei der Reichsversammlung nicht um ein politisches Gesetzgebungsverfahren handelte (siehe Abschn. 5.2.1), ist keineswegs ausgeschlossen, dass aus nichtverfahrensmäßigen Kontexten über eine Organisationsgründung entschieden wird, die den Rahmen für ein ausdifferenziertes Rechtsprechungsverfahren setzt. Würde dies ausgeschlossen, könnten sich Verfahren und Organisationen nur abgeleitet aus bereits bestehenden Organisationen in bereits (vollständig) funktional ausdifferenzierten Gesellschaften bilden; Fragen nach der Entstehung von Sozialformen jenseits der „Moderne“ wären damit wie bisher ausgeklammert.

${ }^{10}$ Ursprünglich wurde das RKG in der Gerichtsordnung von 1495 unter ,camergericht“ oder „unser kgl. oder ksl. Camergericht“ geführt (Jahns 2011, 42). Die Bezeichnung Reichskammergericht wird in den Quellen erst nach dem Westfälischen Frieden von 1648 verwendet und dies ,quantitativ nur in geringem Maße und praktisch nie in offiziellen Dokumenten sowie nur selten in der Kameralliteratur" (ebd.). Die Bezeichnung Reichskammergericht sowie dessen Kürzel seien damit begriffs- und quellengeschichtlich ,,irreführend“ (ebd.), obgleich sie sich in der RKG-Forschung etabliert haben.
} 
die Zugeständnisse an die Reichsstände rückgängig zu machen, zählen die Bemühungen, das RKG zu schließen, es an den Herrscherhof zu verlegen oder die Wahl des Kammerrichters und der Assessoren zu bestimmen. Während der Reformation gelang es beispielsweise Karl V., das RKG kurzzeitig zum ,kaiserlichen Gericht zu machen, indem er alle Richterstellen mit von ihm ausgewählten Personen besetzte“ (Baumann 2019a, 333). Diese Konstellation endete jedoch mit dem Augsburger Religionsfrieden. Die zweite Hälfte des 16. Jahrhunderts wird in der historischen Forschung dagegen als Hochphase des RKG verstanden, in der das RKG seinen satzungsgemäßen Ansprüchen - wie der Durchführung jährlicher Arbeitskontrollen in Form sogenannter Visitationen - weitestgehend nachkam (vgl. Hausmann 1995, 9 ff.; 2003, 147, 157 ff.; Baumann 2001, 2003).

Einige Strukturaspekte, mit denen sich die Rechtsprechung am RKG von denen vorangegangener Gerichte unterschied, sollen hier vor der tieferen organisationssoziologischen Betrachtung erwähnt werden: Mit der bereits 1486 formulierten Bestimmung, das höchste Reichsgericht örtlich vom Reichsoberhaupt zu lösen, unterschied sich das RKG von den Zentralgerichten im spätmittelalterlichen und frühneuzeitlichen England und Frankreich, die ihren Sitz weiterhin an den Residenzen der Königshöfe hatten. Die Rechtsprechung sollte am RKG zwar weiterhin gemäß mittelalterlicher Tradition im Namen des obersten Herrschers ausgeübt werden, jedoch unabhängig von seiner Person und Residenz sein. ${ }^{11}$

Zudem war der Zuständigkeitsbereich des RKG im Vergleich zum Königlichen Kammergericht erweitert worden. So wurde dem RKG eine mehrfache Kompetenz - als erstinstanzliches Gericht ebenso wie als Berufungsinstanz - zugewiesen, die sich im Wesentlichen in vier Bereiche gliedert: In erster Instanz war das RKG zuständig für Zivilprozesse gegen Reichsunmittelbare ${ }^{12}$. Dadurch konnten Reichsunmittelbare gegeneinander oder Untertanen gegen reichsunmittelbare Obrigkeiten klagen (zu den Untertanenprozessen siehe Abschn. 6.2.4 sowie Oestmann 2009a). Zweitens wurde das RKG bei Fällen von Landfriedensbruch tätig. Drittens besaß das RKG eine zivilrechtliche Zuständigkeit als höchste

\footnotetext{
${ }^{11}$ Diese physische Abgrenzung begründete eine dauerhafte Tradition: Auch heute noch wird die oberste Gerichtsbarkeit in Deutschland vom Regierungssitz räumlich getrennt (vgl. Oestmann 2005, 164).

${ }^{12}$ Von der Reichsunmittelbarkeit ist die Reichsstandschaft zu unterscheiden. Letztere bezeichnete den engeren Kreis jener reichsunmittelbaren Glieder, die Sitze und Stimme auf dem Reichstag innehatten. Bis in das 16. Jahrhundert hinein war allerdings vor allem für Grafen, Ritter und Städte vielfach unentschieden, wer zu welcher Gruppe zählte. In der frühneuzeitlichen stratifizierten Gesellschaft herrschte damit im doppelten Sinne eine Stufenfolge von Obrigkeiten vor: Landsässige oder mediate Stände standen zu Kaiser und Reich nur in einem mittelbaren Verhältnis (vgl. Stollberg-Rilinger 2006, 15 f.).
} 
Appellationsinstanz gegen Entscheidungen partikularer Gerichtsbarkeit. Diese galt insbesondere für Urteile der territorialen oder reichsstädtischen Obergerichte sowie der kaiserlichen Hof- und Landesgerichte, bei denen der Beklagte reichsmittelbar war, d. h. der Landeshoheit eines Fürsten unterstand. Die Kompetenzen des RKG als Berufungsgericht für reichsmittelbare Beklagte wurden durch unterschiedliche Ausnahmeregelungen - durch sogenannte privilegia de non appellando - eingeschränkt, die der Kaiser bestimmten Landesherren und Reichsstädten verlieh. Schließlich bestand eine zivilrechtliche Zuständigkeit im Falle von „Rechtsverweigerung“ oder „Rechtsverzögerung“ (Oestmann 2010) durch ein vorinstanzliches Untergericht sowie bei Beschwerden gegen Nichtigkeit - und dies auch gegen Reichsmittelbare, denen Ausnahmen bzw. Appellationsprivilegien gewährt wurden. Bei Nichtbefolgung seiner Urteile konnte das RKG auf Antrag der obsiegenden Partei Strafen aussprechen, diese aber nicht vollstrecken. Derjenige, der ein Urteil des RKG erlangt hatte, musste vielmehr bei territorialen Herrschaftsträgern bzw. bei den 1512 neu geschaffenen Reichskreisen die Durchsetzung beantragen (vgl. Diestelkamp 1995b, 91-124; Baumann 2019a, 333; Jahns 2011, 78-81; Dick 1981, 69 f.).

Neben dem skizzierten Problemdruck einer fehlenden ständigen Rechtsprechung im Alten Reich, lässt sich die Annahme einer originären Organisationsbildung ergänzend ex negativo erschließen, indem gefragt wird, inwiefern die Gründung des Gerichts auf andere Organisationen und deren Folgeprobleme zugerechnet werden kann. Wie oben gezeigt, lassen die zentralen Entscheidungskontexte der Rechtsetzung, Rechtsprechung und Rechtsdurchsetzung vor der Gründung des RKG zwar keine organisations- und verfahrensförmige Ausdifferenzierungen erkennen (siehe Abschn. 5.2). Allerdings finden sich in der Gerichtsordnung des RKG einzelne Strukturaspekte, die sich bereits in angrenzenden Bereichen und Einrichtungen der vormodernen Gesellschaft ausgebildet haben. Dazu zählen das genannte Prinzip der Stellvertretung durch Gelehrte, die Beratungen hinter verschlossenen Türen oder die Einrichtung von Visitationen. Angesprochen sind damit Strukturen, die beispielsweise auf die Runde geistlicher 
Gelehrter im Kreis des Papstes - der sogenannten Sacra Rota Romana ${ }^{13}$ - zurückgehen. Dieser selektive Bezug auf die 300 Jahre zuvor eingeführte Rota kann nach dem hier verfolgten Zugang eines allgemeinen Systembegriffs von Organisation jedoch noch keine ausreichende Begründung für eine abgeleitete Genese des RKG geben. Denn: Eine abgeleitete Organisationsbildung - wenn man die Unterscheidung als scharfe Trennung ernst nimmt - würde voraussetzen, dass es sich bei der Rota bereits um eine Organisation im hier verstandenen systemtheoretischen Sinne handelte. Ohne die dazu empirisch notwendigen Analysen vornehmen zu können, ist einzurechnen, dass in den hierzu gesichteten Forschungen keine Hinweise auf die Organisiertheit römischer Gerichte entnommen werden konnten. Für die Vermutung einer originären Organisationsbildung spricht auch, dass nicht erkennbar ist, inwiefern die Gründung des RKG Strukturprobleme römischer Gerichte löste bzw. dass das RKG Folgen dieser bearbeitete. Das RKG war, zugespitzt formuliert, kein Auffangbecken für geistliche Rechtsgelehrte.

Gegen die Annahme einer abgeleiteten Organisationsbildung kann darüber hinaus in Anschlag gebracht werden, dass die oben nur kursorisch behandelten Verwaltungs- und Rechtstätigkeiten in der Zeit nach schichtspezifischen Kriterien vergeben wurden. Dass das Richteramt ein Laienamt war, zeigt sich auch darin, dass eine Tätigkeit als Richter oder Anwalt nicht das Ziel eines juristischen Studiums war; "dafür fehlten die prozessrechtlichen Voraussetzungen“ (Scheurmann 1994, 119 f.). Ausgebildete Gelehrte standen bis zum Ende des 15. Jahrhunderts vor allem im Dienst der Kirche und beförderten an geistlichen Gerichten die Anwendung des spätrömischen Rechts. Für weltliche Gerichte vollzog sich der Wechsel vom patrizischen Schöffen zum studierten Juristen im 16. Jahrhundert, als sich der genossenschaftliche Verband Universität zu einer Lehrorganisation entwickelte. Die Zugehörigkeit zum Rechtskreis einer Universität

\footnotetext{
${ }^{13}$ Die als Rota Romana bezeichnete ,Runde“ an geistlichen Richtern bildete im 11. und 12. Jahrhundert zunächst keinen eigenen Gerichtshof, sondern war Teil der römischen Kurie. Sie war aus der Praxis entstanden, dass der Papst mit zunehmender Anzahl der Rechtsstreitigkeiten bestimmte Rechtssachen von Fall zu Fall einem Auditor - einer gerichtserfahrenen Person - zur Vorbereitung eines Urteils übertrug. Gemäß dem römisch-kanonischen Prozessrecht mussten die Auditoren dabei vor jeder Entscheidung auch den Rat rechtskundiger Gelehrter einholen. Auf diese Weise entstand ein Kollegium ständiger Auditoren mit eigenen Prozessgewohnheiten. Außerdem orientierten sich die Auditoren der Rota an den von ihnen gefällten Entscheidungen. Für die Aufnahme in das Auditoren-Gremium musste zudem eine fachliche Prüfung bestanden werden, die in der Disputation über einen Rechtsfall bestand. Im Unterschied zum erwähnten Hofrichter musste ein Auditor sich durch Sittenreinheit auszeichnen und das Doktorat in beiden Rechten erlangt haben. Seine Verpflichtung wurde mit einem Eid besiegelt (vgl. Becker 2007, 1-18). Für den Hinweis auf die Sacra Rota Romana danke ich Peter Oestmann.
} 
schloss die Ernennung zum Richter bzw. Urteiler einer Stadt bis dahin aus. Bürger der Stadt waren umgekehrt nicht zur Universität zugelassen (vgl. Schmutz 2000, 73 f.; Müßig 2007, 29 f.; Jahns 2011, 184; Oestmann 2011, 99 f.; Arlinghaus 2018, 36 ff.). ${ }^{14}$ Offenbar fand erst nach der Reformation mit der zunehmenden Gründung von Universitäten durch Landesherren ein Wechsel des Primats von kirchlich-religiösen hin zu politischen Abhängigkeiten statt. Die Fortbildung in römisch-kanonischen Rechten oblag danach nicht mehr allein der Kirche. So wurde etwa die Rechtsschule in Bologna später eine beliebte Ausbildungsstätte für eine Anstellung am RKG (vgl. Stichweh 1991, 47, 307 f., 352 ff.; Füssel 2020, 262, 278; Baumann 2011, 365 ff.).

Vor diesem Hintergrund lässt sich nicht erkennen, dass mit der Gründung des RKG bestimmte Strukturprobleme der universitären Rechtslehre oder der akademischen Gerichtsbarkeiten gelöst worden wären. ${ }^{15}$ Die Einrichtung einer ständigen Adresse für höchste Rechtsentscheidungen und die dazu verabschiedete Personalordnung schufen erst einen Bedarf an juristisch ausgebildetem Dauerpersonal jenseits der weitgehend immobilen ständisch-kirchlichen Dienstverhältnisse. Die Gründung des RKG kann deshalb nicht primär als eine Strukturangleichung durch Zwang, Druck oder Imitation (vgl. Meyer/Rowan 1977) an eine gesellschaftliche Organisationsumwelt erklärt werden.

Die Gerichtsordnungen des RKG waren allerdings umgekehrt maßgebend für die Rezeption des römischen Gelehrtenrechts an weltlichen Gerichten und für

\footnotetext{
${ }^{14}$ Hinsichtlich der Höchstgerichtsbarkeit in Frankreich gab es seit Beginn des 15. Jahrhunderts Klagen über unzureichend gebildete Prozessvertreter und Richter. Eine universitäre Ausbildung im römisch-kanonischen Recht wird für Anwälte wie für Richter ,erst in der Frühen Neuzeit vorgeschrieben“ (Müßig 2007, 30). Für Frankreich stellt die Rechtshistorikerin heraus, dass erst ,,vor dem Parlement de Paris ein Vertretungsmonopol vereidigter und zugelassener Personen festgelegt wurde [...] und erst dort entsteht das Bedürfnis nach Personen, die mit dem stilus curiae und dem modus advocandi vertraut sind“" (ebd., 30, Herv. i.O.).

${ }^{15}$ Über die mittelalterliche Genese der akademischen Gerichtsbarkeit im Reich herrscht in der Forschung bis in die Gegenwart weitgehende Uneinigkeit. Zwar wird immer wieder auf die authentica habita von 1158 und die Tradition der Universität Bologna verwiesen. Mit dieser Referenz ist aber nur unzureichend erklärt, ,wie die deutschen, eher am Modell der Pariser Universität orientierten Gründungen diese übernahmen“ (Füssel 2020, 264). Gerade Paris habe nur über eine sehr eingeschränkte Gerichtsbarkeit verfügt. Jenseits des langewährenden Streits um die Entstehung aus geistlichen (Papst) und weltlichen (Kaiser) beziehungsweise kirchlichen oder staatlichen Wurzeln wird von Marian Füssel der Aspekt der Herleitung aus der korporativen Autonomie der Universitäten betont. Genossenschaft und Herrschaft bildeten offenbar keinen Widerspruch, sondern ergänzten einander (vgl. Füssel 2020, 262, 278).
} 
die damit einhergehende Professionalisierung und Vereinheitlichung der Rechtsprechung im Reich; sie werden in der historischen Forschung denn auch als Vorbilder bezeichnet. Die rechtshistorischen Verweise auf die Ausstrahlungswirkungen der RKG-Ordnungen (siehe z. B. Oestmann 2013, 17) können aus dieser Perspektive als Anhaltspunkte für abgeleitete Gerichtsgründungen und Reformen in den einzelnen Territorien gelesen werden. ${ }^{16}$ Wenn dabei von der Gründung des RKG ein wesentlicher Impuls für die Ausbildung universitär ausgebildeter Juristen und entsprechender Rechtskenntnisse abgeleitet wird, ist zugleich zu berücksichtigen, dass sich die genannten gesellschaftsstrukturellen Entwicklungen im Einzelnen unterschiedlich bedingten und im zeitlichen Verlauf von vielfältigen Brüchen auszugehen ist. Gegenüber dem problematisierten komplementären Bedingungs- bzw. Steigerungsverhältnis von organisatorischer und gesellschaftlicher Differenzierung wurde an dieser Stelle deshalb versucht, die historischen Konstellationen und Tendenzen, die für eine originäre Organisationsbildung sprechen, zu konkretisieren.

\footnotetext{
${ }^{16}$ Die Tatsache, dass auch nach der Gründung des RKG weiterhin gerichtliche Anfragen direkt an den König bzw. Kaiser gerichtet wurden, zog spätestens 1559 die Einrichtung des Reichshofrats (RHR) nach sich. Das RKG und der kaiserliche RHR in Wien ergänzten ihre Zuständigkeitsbereiche in vielen Hinsichten; sie standen aber auch in gewisser Konkurrenz. Der RHR genoss zudem in einigen Streitfällen eine exklusive Kompetenz. In dieser Arbeit findet der RHR nur punktuell mit Blick auf die primäre Fragestellung Erwähnung. Zum Verständnis seien einige zentrale Aspekte zum RHR erwähnt, die die einleitend begründete Wahl des RKG als Gegenstand einer historisch-soziologischen Organisationsanalyse ergänzen. Zum einen wurde der RHR wegen seiner geringeren bzw. verzögerten Aktenverzeichnung bislang vergleichsweise wenig erforscht. Zum anderen war der RHR wie das Reichshofgericht an die Person und den Aufenthaltsort des Monarchen gebunden. Beim RHR handelte es sich dabei um ein allgemeines, beratendes Gremium, das neben der kaiserlichen Rechtsprechungsgewalt auch andere Tätigkeiten in dessen Auftrag ausübte - d. h. nicht nur als Gericht im engeren Sinne fungierte. Insbesondere konnte der König bzw. Kaiser mit dem RHR entscheiden, ob er die an ihn gerichteten Fälle selbst mit seinen Beratern entschied oder den Gegenstand an das RKG weiterleitete (vgl. Diestelkamp 1994a, 117; 1999, 206; Schildt 2006, 6-19; Jahns 2011, 74 ff.) Für spezifische Einsichten sei auf die Arbeiten von Wolfgang Sellert (1965, 1999, 2004) und jüngst Thomas Dorfner (2012, 2015a/b) verwiesen.
} 
Open Access Dieses Kapitel wird unter der Creative Commons Namensnennung 4.0 International Lizenz (http://creativecommons.org/licenses/by/4.0/deed.de) veröffentlicht, welche die Nutzung, Vervielfältigung, Bearbeitung, Verbreitung und Wiedergabe in jeglichem Medium und Format erlaubt, sofern Sie den/die ursprünglichen Autor(en) und die Quelle ordnungsgemäß nennen, einen Link zur Creative Commons Lizenz beifügen und angeben, ob Änderungen vorgenommen wurden.

Die in diesem Kapitel enthaltenen Bilder und sonstiges Drittmaterial unterliegen ebenfalls der genannten Creative Commons Lizenz, sofern sich aus der Abbildungslegende nichts anderes ergibt. Sofern das betreffende Material nicht unter der genannten Creative Commons Lizenz steht und die betreffende Handlung nicht nach gesetzlichen Vorschriften erlaubt ist, ist für die oben aufgeführten Weiterverwendungen des Materials die Einwilligung des jeweiligen Rechteinhabers einzuholen.

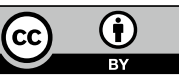

